\title{
PALLIATIVE LARGE BOWEL RESECTIONS. IMPACT OF THE LAPAROSCOPIC APPROACH
}

\author{
Veselin Marinov, Kiril Draganov, Nikolay Katev, Anastazia Petreska, Dimitar Rusenov, \\ Radoslav Gajdarski \\ Department of Miniinvasive and Laparoscopic Surgery, HPB and General Surgery Clinic, \\ Acibadem City Clinic, Tokuda Hospital, Sofia, Bulgaria
}

\begin{abstract}
INTRODUCTION: Nowadays colorectal cancer (CRC) incidence rate increases in the Western world. The lack of effective screening programs results in diagnosis of advanced cases in our country. Combination of hematogenic, peritoneal or systemic CRC dissemination with present or potential complications from local disease is not an exception. The decision for palliative resection is not easy and is a matter of balance between potential risks of operative intervention and advances due to local tumour resection. Positive aspects of the laparoscopic approach are important in planning of these interventions. The aim of this study is to assess the indications for laparoscopic palliative large bowel resections in the literature available and to share our own experience.
\end{abstract}

PATIENTS AND METHODS: During a two-year period, in the HPB and General Surgery Clinic, Acibadem City Clinic, Tokuda Hospital of Sofia, six laparoscopic palliative colon resections were performed. In the all cases, multiple liver metastases were diagnosed without possibility of liver resection. The early perioperative results were analyzed.

RESULTS: Four left colon resections were performed with primary anastomosis due to high-level large bowel obstruction and two right colectomies were done for primary tumour bleeding. Three patients were over 77 years old and presented with comorbidity. Average hospital stay was 5,6 days. No perioperative complications were observed.

CONCLUSION: Minimal surgical trauma, short recovery period and well-defined indications are important features in the process of planning of palliative large bowel resections in patients with disseminated CRC. The objective is to reduce risks of complications connected with primary tumour and to assure a good patient's quality of life. Scr Sci Med 2017; 49(3): 27-30

Keywords: laparoscopic surgery, colorectal cancer, large bowel, palliative resection, tumour bleeding

\footnotetext{
Address for correspondence:

Veselin Marinov

HPB and General Surgery Clinic,

Acibadem City Clinic,

Tokuda Hospital,

Sofia, Bulgaria

$51 B$ Nikola Vaptsarov Blvd, 1407 Sofia

e-mail:vesika_m@mail.bg
}

Received: April 10, 2017

Accepted: July 3, 2017

\section{INTRODUCTION}

Lack of adequate screening programs and late diagnostics are the main reasons for the high incidence of advanced forms of colorectal cancer (CRC) in Bulgaria. The presence of multiple liver metastases, peritoneal carcinosis, or distant hematogenic involvement are not exceptions. Considering the fact that CRC occurs very often in older patients with associated comorbidities, the debate on the treatment of these patients is imperative. The complications as- 
sociated with the local enlargement of the malignant process are frequent and significantly impair the quality of life (QoL) of metastatic CRC patients. The most common symptoms are related to chronic hemorrhage and the development of anemic syndrome, pain, and risk of obstruction.

Making a decision to perform palliative resection of the colon is difficult and should be considered after a very careful and detailed discussion with the patient and his relatives. The advantages of the laparoscopic approach in these cases are of paramount importance. Short hospital stays, rapid recovery, low pain levels, peritoneal cavity exploitation are arguments in favour of a cytoreductive surgical intervention aimed at the primary fat-free process to address existing or potential complications.

The purpose of this communication is to discuss the indications for conducting laparoscopic palliative colon resection, to share our initial experience and to review of literature data available.

\section{PATIENTS AND METHODS}

During a two-year period, in the HPB and General Surgery Clinic, Acibadem City Clinic, Tokuda Hospital of Sofia, six laparoscopic palliative colon resections in patients with hematogenically disseminated CRC and multiple hepatic metastases without any possibility of liver resection were performed. A retrospective analysis of the cases was carried out, and perioperative indicators were discussed.

\section{RESULTS}

In all the cases, CRC was associated with multiple non-resectable liver metastases. At admission, the patients presented with a preserved liver function, with no evidence of hepatic insufficiency. There were four males and two females. The patients aged over 70 years prevailed as three of them were between 77 and 78 years of age. Mean age was 67,5 years.

In four patients, the primary process was localized in the sigmoid colon. One patient presented with locally advancing T4 tumour engaging the muscularaponeurotic layers of the anterior abdominal wall. CRC was localized in the right colon in two patients.

As an indication for surgical treatment, we assumed the presence of anemic syndrome in four patients (Hb of 114-68 g/L) accompanied by astheniaadynamic complaints, pain and varying degrees of sigmoid colon stenosis, however, without any clinical manifestation of intestinal obstruction.

With all the candidates for palliative surgery, a thorough conversation between the treating physician, the patient and his or her relatives was carried out. The nature of the disease, its stage, the risks of complications associated with the enlargement of the primary pathologic process, the prognosis, the possibilities for adjuvant therapy, the type of planned laparoscopic surgery, the risks and benefits of its implementation were explained.

Four laparoscopic resections of sigmoid colon, rectum-sigmoid colon with primary anastomosis and two right hemicolectomies with extracorporeal anastomosis were performed.

In all the cases, excisional liver biopsies were done. No additional ports were required.

The surgical technique itself was not different from that of radical resections. We preferred a medial approach with identification of colon vessels and their clipping with polymer clips at baseline, standard regional lymphadectomy as well as compliance with anatomical dissection plans. In the case of the locally advanced process of the sigmoid colon, excision of the musculo-aponeurotic layers of the abdominal wall was performed in ablative ranges.

The average duration of the interventions was 156 minutes. The average blood loss was $63 \mathrm{~mL}$. No conversion to open surgery was required at all.

Due to the lack of statistical validity of the group of six patients only, there was no comparative analysis of liver biopsy time prolongation.

On a first post-operative day, patients were set up straight, extracting the nasogastric tubes and providing oral intake of fluids. Usually, we fed the patients after two 2 postoperative days. Average hospital stay was 5,6 days. No complications were observed in the postoperative period.

\section{DISCUSSION}

CRC remains one of the most common oncological diseases on a global scale with more than 1.2 million new cases per year. Approximately half of these patients will die from the disease within five years of diagnosis (1). Approximately $20 \%$ of patients are in stage IV at diagnosis (2). Despite advances in systemic therapy, most patients in this stage are not 
subject to radical surgery and thus undergo palliative procedures only. In emergencies, they undergo palliative resection, bypass, stoma, stenting, etc. (3). On the other hand, such patients presenting with asymptomatic disease and a lack of emergency with regard to the primary tumour are debatable about the benefits of possible palliative resection as well as the risks associated with it. The debate on this topic continues in the recent literature available (4-11). According to one theory, in primary tumour resection, tumour burden is reduced and even if the disease advances, systemic therapy remains more effective. Concomitantly, the risk of complications associated with the primary tumour is reduced. The opposite thesis is that, in the absence of CRC symptoms, no time should be lost with surgery and systemic therapy should be initiated as soon as possible.

Some isolated series of patients were already reported comparing the palliative resection performed in conventional and laparoscopic manner. In such a series comparing 18 conventional with 11 laparoscopic operations, early perioperative features completely favoured the laparoscopic approach (12).

According to our opinion, with the development of systemic medication therapy, CRC surgery should be more aggressive. Making a decision to perform palliative laparoscopic surgery should be the result of a serious discussion between the physician, the patient and the patient's relatives.

\section{CONCLUSION}

Based on the comparatively scarce data in the literature available and the statistically non-specific present patient contingent, we can suggest that the laparoscopic approach to palliative CPC resections is accompanied by very good perioperative outcomes and rapid patients' recovery. Obviously, the larger group of patients as well as the possibility of analysis of late postoperative results could answer the question of the appropriateness of such surgical behaviour.

\section{REFERENCES}

1. Brenner H, Kloor M, Pox CP. Colorectal cancer. Lancet. 2014;383(9927):1490-1502. doi: 10.1016/ S0140-6736(13)61649-9.

2. Adam R, de Gramont A, Figueras J, Kokudo N, Kunstlinger F, Loyer E, et al.; of the EGOSLIM (Ex- pert Group on OncoSurgery management of Liver Metastases) group. Managing synchronous liver metastases from colorectal cancer: a multidisciplinary international consensus. Cancer Treat Rev. 2015;41(9):729-41. doi: 10.1016/j.ctrv.2015.06.006.

3. Søreide K. Emergency management of acute obstructed left-sided colon cancer: loops, stents or tubes? Endoscopy. 2013;45(4):247-8. doi: 10.1055/s-0032-1326404.

4. Maeda $Y$, Shinohara T, Katayama T, Minagawa N, Sunahara M, Nagatsu A, et al. A laparoscopic approach is associated with a decreased incidence of SSI in patients undergoing palliative surgery for malignant bowel obstruction. Int J Surg. 2017;42:90-4. doi: 10.1016/j.jijsu.2017.04.052.

5. Tei M, Wakasugi M, Akamatsu H. Short-term outcomes of single-port surgery for palliative resection of the primary tumor in patients with incurable stage IV colon cancer. Asian J Endosc Surg. 2016;9(4):258-64. doi: 10.1111/ases.12289.

6. Biondi A, Vacante M, Ambrosino I, Cristaldi E, Pietrapertosa G, Basile F. Role of surgery for colorectal cancer in the elderly. World J Gastrointest Surg. 2016;8(9):606-13. doi: 10.4240/wjgs.v8.i9.606.

7. Zhou MW, Gu XD, Xiang JB, Chen ZY. Clinical safety and outcomes of laparoscopic surgery versus open surgery for palliative resection of primary tumors in patients with stage IV colorectal cancer: a meta-analysis. Surg Endosc. 2016;30(5):1902-10. doi: 10.1007/s00464-015-4409-1.

8. Patel S, Chang GJ. Primary tumor resection in metastatic colorectal cancer: Please pass the salt. JAMA Oncol. 2015;1(9):1213-4. doi: 10.1001/ jamaoncol.2015.2757.

9. Chang GJ. Primary tumor resection in stage IV colorectal cancer: the debate continues. Dis Colon Rectum. 2011;54(8):919-20. doi: 10.1097/ DCR.0b013e31821ccf05.

10. Mik M, Dziki L, Galbfach P, Trzcinski R, Sygut A, Dziki A. Resection of the primary tumour or other palliative procedures in incurable stage IV colorectal cancer patients? Colorectal Dis. 2010;12(7 Online):e61-7. doi: 10.1111/j.1463-1318.2009.01860.x.

11. Cellini C, Hunt SR, Fleshman JW, Birnbaum EH, Bierhals AJ, Mutch MG. Stage IV rectal cancer with liver metastases: is there a benefit to resection of the primary tumor? World J Surg. 2010;34(5):11028. doi: $10.1007 / \mathrm{s} 00268-010-0483-7$. 
Palliative Large Bowel Resections. Impact of the Laparoscopic Approach

12. Akagi T, Inomata $M$, Etoh $T$, Yasuda $K$, Shiraishi N, Kitano S. Laparoscopic versus conventional palliative resection for incurable, symptomatic stage IV colorectal cancer: impact on short-term results. Surg Laparosc Endosc Percutan Tech. 2011;21(3):184-7. doi: 10.1097/ SLE.0b013e31821db75e. 